\title{
Multidrug resistant tuberculosis
}

\author{
James Millard clinical lecturer in global health ${ }^{1}$, Cesar Ugarte-Gil epidemiologist ${ }^{2}$, David A J Moore \\ professor of infectious diseases \& tropical medicine ${ }^{3}$
}

${ }^{1}$ Brighton and Sussex Medical School, Brighton, UK; ${ }^{2}$ Instituto de Medicina Tropical Alexander von Humboldt, Universidad Peruana Cayetano Heredia, Lima, Peru; Department of International Health, Johns Hopkins Bloomberg School of Public Health, Baltimore, USA; ${ }^{3}$ TB Centre, London School of Hygiene and Tropical Medicine, London WC1E 7HT, UK

Tuberculosis remains a major cause of morbidity and mortality globally, with an estimated nine million people developing the disease and 1.5 million deaths in 2013; equating to 4100 deaths a day. ${ }^{1}$ Nevertheless, considerable gains have been made in international tuberculosis control; incidence rates are decreasing (albeit slowly) and mortality has been reduced by $45 \%$ worldwide. The advent of multidrug resistant tuberculosis threatens this progress. In this review we detail the challenges faced globally in the diagnosis, treatment, and control of multidrug resistant tuberculosis and why this matters to high and low burden multidrug resistant tuberculosis settings alike.

\section{What is multidrug resistant tuberculosis?}

Rifampicin and isoniazid form the backbone of conventional first line treatment for tuberculosis. Multidrug resistant tuberculosis refers to tuberculosis that is resistant to both rifampicin and isoniazid. This classification is important because treatment outcomes for this group of patients are far worse than for patients with fully drug susceptible or lesser forms of drug resistant tuberculosis. ${ }^{12}$ Multidrug resistant tuberculosis with additional resistance to any fluoroquinolone (such as ofloxacin or moxifloxacin) and also any one of the three second line injectable agents (amikacin, capreomycin, kanamycin) is designated as extensively drug resistant tuberculosis.

\section{How common is it?}

"Missing" patients are a major problem in assessing the burden of tuberculosis globally. ${ }^{1}$ Without access to universal drug susceptibility testing, national tuberculosis programmes have to estimate burden by extrapolation from periodic national or subnational surveys. This generates the global estimate of 480 000 cases of multidrug resistant tuberculosis in 2013 (range 350 000-610 000), of which $9 \%$ have extensively drug resistant tuberculosis. Out of the estimated 480000 cases of multidrug resistant tuberculosis in 2013, only 136000 were reported to the World Health Organization. ${ }^{1}$ Globally, the main barrier to diagnosis is the lack of access to drug susceptibility testing in quality assured laboratories. Even among bacteriologically confirmed cases of tuberculosis, few were tested for multidrug resistant tuberculosis, with particularly low levels in the African and South East Asia regions. ${ }^{1}$ In the United Kingdom, where all patients with tuberculosis undergo drug susceptibility testing and ascertainment is therefore believed to be almost complete, the reported number of cases remains small but increased from 28 to 81 per year from 2000 to $2012 .^{3}$

\section{What is the prognosis?}

An estimated 210000 deaths (range 130 000-290 000) occur from multidrug resistant tuberculosis worldwide each year, as a consequence of never being diagnosed, the gap between diagnosis and treatment, and poor treatment outcomes. Worldwide, fewer than half of cases achieve treatment success. ${ }^{1}$ In the United Kingdom more than $70 \%$ of patients with multidrug resistant tuberculosis achieve treatment success, but it has been shown that it is possible to achieve comparable (or even better) outcomes, even in low income settings. ${ }^{4}$

\section{Who is at risk of multidrug resistant tuberculosis?}

All patients with tuberculosis should undergo drug susceptibility testing because only testing those perceived to be at risk will miss up to half of multidrug resistant cases. ${ }^{1}$ Nevertheless, it is clear that multidrug resistant tuberculosis is more common in some groups, such as the former prison inmate in the example (box 1). In all settings patients who have previously been treated for tuberculosis are at higher risk for multidrug resistant tuberculosis, with recent estimates suggesting that $3.5 \%$ of new cases and $20.5 \%$ of previously treated cases have multidrug resistant tuberculosis. ${ }^{1}$ However, rates of multidrug resistant tuberculosis vary considerably between countries and regions (table $\Downarrow$ ) with 27 high burden countries accounting for more than $85 \%$ of cases. Worldwide, China, India, and the Russian Federation contribute the largest total number of cases. However, the highest proportions are found in eastern Europe and Central Asia, with around $20 \%$ of new cases and $50 \%$ of previously treated cases having multidrug resistant tuberculosis. In countries with a high burden of tuberculosis such as South Africa, even 


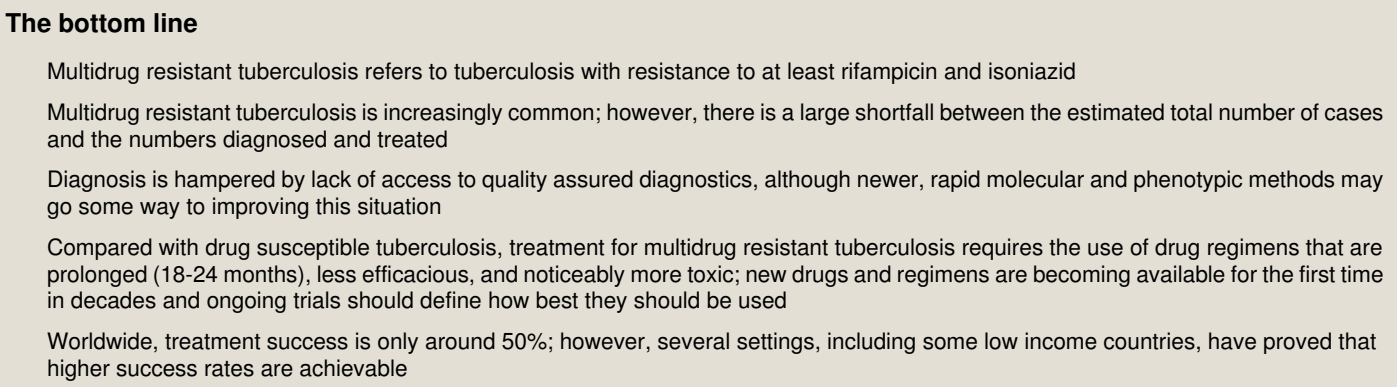

Compared with drug susceptible tuberculosis, treatment for multidrug resistant tuberculosis requires the use of drug regimens that are prolonged (18-24 months), less efficacious, and noticeably more toxic; new drugs and regimens are becoming available for the first time in decades and ongoing trials should define how best they should be used

Worldwide, treatment success is only around $50 \%$; however, several settings, including some low income countries, have proved that higher success rates are achievable

\section{Sources and selection criteria}

We searched Medline, Embase, and Scielo with the terms "MDR TB", "Tuberculosis, Multidrug-Resistant". We also reviewed the World Health Organization Global Tuberculosis Report 2014 and other national tuberculosis programme reports. We included data from systematic reviews/meta-analyses, randomised controlled trials, and good quality observational studies focused on multidrug resistant tuberculosis.

a relatively low percentage of multidrug resistant tuberculosis among the large number of cases translates into a high incidence rate when expressed as cases of multidrug resistant tuberculosis per 100000 population per year.

In the United Kingdom $1.4 \%$ of new and $5.7 \%$ of previously treated patients have multidrug resistant tuberculosis, with $89 \%$ of these patients being born outside the United Kingdom (45\% from South Asia, 19\% from eastern Europe and 19\% from Sub-Saharan Africa). ${ }^{5}$ The proportion of multidrug resistant tuberculosis in UK cases of tuberculosis in 2013 did not exceed $6 \%$ in most groups. Strikingly, however, for people born in eastern Europe it was over $24 \%{ }^{4}$

Once drug resistance develops the possibility of transmission to other people emerges, with up to half of multidrug resistant tuberculosis cases arising in this way. ${ }^{1367} \mathrm{In}$ addition, in high incidence settings where reinfection is common, an unknown fraction of previously treated people with tuberculosis will have been infected by multidrug resistant tuberculosis unrelated to their previous infection and thus transmission rates are likely to be systematically underestimated. Other risk factors for multidrug resistant tuberculosis may include being a household contact of a known infected person, younger age, and (putatively) the strain type of tuberculosis. ${ }^{89}$

\section{How is it diagnosed?}

The diagnosis of multidrug resistant tuberculosis is based on the detection of resistance to rifampicin and isoniazid in the causative organism in a clinical sample, usually sputum. The quality and quantity of the sputum sample can affect test performance. ${ }^{10}$ An increasing array of new tools for the diagnosis of multidrug resistance has become available in recent years, for the detection of either phenotypic (growth based) or genotypic (molecular markers) resistance. The use of specific tests in different regions is heterogeneous and most regions will use several different tests in combination.

\section{Phenotypic indirect drug susceptibility testing}

Conventional phenotypic tests use methods of indirect drug susceptibility testing. This requires an isolate to be cultured first and then inoculated into solid or liquid media. This leads to a considerable delay until results are available, often several months, therefore results are often not available in a clinically useful timeframe. Automated systems such as the Mycobacteria Growth Indicator Tube 960 (MGIT 960, Becton Dickinson Microbiology Systems, Sparks, MD) speed up culture times through the use of liquid media and automated sensing of culture positivity, but still require subsequent indirect sensitivity testing. High throughput is a major advantage although tempered by relatively high cost and contamination rates.

\section{Phenotypic direct drug susceptibility testing}

Phenotypic direct drug susceptibility testing delivers a result direct from inoculation of the sputum sample thus does not need to be subcultured. Samples are inoculated into media containing drugs at specific critical concentrations such that the presence of growth (whether detected by a colour change or visual identification of microscopic growth) is indicative of phenotypic resistance. ${ }^{11}{ }^{12}$ These methods are rapid, non-commercial, and low cost making them well suited to resource constrained settings with a high burden of tuberculosis. ${ }^{13}$ Tests endorsed by WHO include microscopic observation drug susceptibility (MODS) and the nitrate reductase assay.

\section{Genotypic drug susceptibility testing}

Genotypic methods test for the presence of known resistance mutations associated with multidrug resistant tuberculosis. Two types of geneotypic assay have been endorsed by WHO: molecular beacon assays and line probe hybridisation assays.

WHO has endorsed the use of the molecular beacon assay Xpert MTB/RIF (Cepheid, Sunnyvale, CA) as an initial diagnostic test where multidrug resistant tuberculosis is common. ${ }^{14}$ This test is a real time polymerase chain reaction that detects Mycobacterium tuberculosis and rifampicin resistance (by identification of mutations in the mycobacterial rpoB gene). It is performed directly on sputum, with a turnaround time of two hours, which is a key advantage. Identification of resistance to rifampicin still necessitates subsequent and more extensive drug susceptibility testing of other first line and second line agents, usually by phenotypic (culture based) methods. There are concerns about false negative and false positives test results for rifampicin resistance ${ }^{15}$ and the increased frequency of mixed infections in high prevalence settings,${ }^{16}$ so local evaluation of multidrug resistant tuberculosis epidemiology before rollout may be prudent. The higher cost of the test compared with phenotypic testing is also a constraint, particularly since isoniazid susceptibility testing is not assessed. Costs range from $\$ 10$ (£6; €9) (for low income countries) for each test, to more than $\$ 70 .{ }^{17} 18$

An alternative molecular test platform endorsed by WHO is the line probe assay. ${ }^{19}$ This assay exists in two main commercial forms - the INNO-LipA Rif.TB (Fujirebio Europe, Ghent, 


\section{Box 1 An example of how multidrug resistant tuberculosis might present}

A 40 year old man, originally from the Russian Federation and now resident in London, presented to the emergency department of his local hospital with a history of dry cough, fever, and night sweats for several months. His medical history was unremarkable and he had never previously received treatment for tuberculosis. He had spent two years in a Russian prison three years previously. His chest radiograph revealed patchy shadowing in the right upper lobe with several cavities. Sputum was positive for acid fast bacilli and he was prescribed standard first line quadruple tuberculosis treatment as an outpatient, with a combination of rifampicin and isoniazid, pyrazinamide, and ethambutol. Despite this, his symptoms remained and five weeks later the results of a sputum culture confirmed Mycobacterium tuberculosis with resistance to rifampicin and isoniazid.

Belgium), which detects rifampicin resistance, and Genotype MTBDRplus (Hain Lifescience, Nehren, Germany), which identifies rifampicin (through detection of mutations in the rpoB gene) and isoniazid resistance (mutations in the kat $G$ and $i n h A$ genes). ${ }^{20}$ It is performed on cultured isolates or on smear positive sputum samples, ${ }^{21}$ and a version is now available (genotype MTBDRsl), although not yet endorsed by WHO, for fluoroquinolone, aminoglycoside, and ethambutol drug susceptibility testing. ${ }^{22}$

As whole genome sequencing becomes increasingly affordable and accessible, the horizon holds potential promise of a single technology that could be utilised simultaneously to detect genotypic resistance to rifampicin and isoniazid and key second line drugs-although some work is needed to characterise a full library of resistance conferring mutations. A useful byproduct of sequencing in this way would be the molecular epidemiological insights yielded into the contribution of transmission (as opposed to reactivation of latent multidrug resistant tuberculosis infection) in different settings. ${ }^{23}$

\section{How is it treated?}

Of the 136000 patients with multidrug resistant tuberculosis reported in 2013, just over $71 \%$ (97 000) began treatment. ${ }^{1}$ This treatment gap is heterogeneous between countries, with fewer than half of eligible patients enrolled in treatment in the African region. However, these numbers seem to represent progress, particularly in India, Russia, and South Africa, with a more than $150 \%$ increase in enrolment to treatment since 2009. ${ }^{1}$ Achieving the goal of universal access to the diagnosis and treatment of multidrug resistant tuberculosis requires the strengthening of health services in general. Simply improving rates of treatment initiation in a poor programme is likely to be ineffective and may increase rates of drug resistance and promote transmission. ${ }^{24}$

\section{Recommended drug regimens}

Currently recommended treatment for multidrug resistant tuberculosis requires 18-24 months of at least five drugs, none of which are as potent as rifampicin or isoniazid and all of which are more toxic and less well tolerated. Countries with access to quality assured drug susceptibility testing for second line drugs are likely to offer an "individualised" regimen tailored to a patient's resistance pattern. Countries without this facility are likely to offer a "standardised" regimen, which is broadly similar for all patients and based on historical resistance patterns and which drugs have been used in that region. Even in the context of individualised regimens, initial treatment is often empirical (and based on similar principles to standardised treatment), pending second line drug susceptibility testing. ${ }^{25}$

Multidrug resistant tuberculosis should be treated with an injectable agent such as amikacin, kanamycin, or capreomycin (streptomycin is usually not used because of high rates of resistance), a fluoroquinolone (levofloxacin, moxifloxacin, or gatifloxacin are recommended), and at least three other agents with probable activity (ethionamide or prothionamide, cycloserine, para-aminosalicylic acid). First line agents (that is, pyrazindamide and ethambutol) with retained activity can also be used.

Ideally the injectable agent is administered daily for the first 6-8 months, forming an "intensive phase" of treatment, with the other drugs then continued, forming a "continuation phase." 25 In practice, adverse effects often supervene (see example in box 2) and require stopping the injectable agent, managing side effects or swapping drugs for remaining alternatives.

\section{Challenges to management}

Managing the side effects of treatment forms an important part of management and a failure to do so can affect adherence and drive the development of further resistance. Particularly common side effects include local injection site complications, ototoxicity, and nephrotoxicity from the injectable agents; neuropsychiatric symptoms and seizures with cycloserine; and diarrhoea with para-aminosalicylate sodium. Hypothyroidism can occur with ethionamide or prothionamide or para-aminosalicylic acid. Drug induced hepatitis is not uncommon, particularly in association with pyrazinamide. Delivery and monitoring of the drug regimens is complex, and drug stockouts are common and undermine efforts. All regimens are based on cohort data and expert opinion, with a conspicuous absence of randomised controlled trial evidence to inform treatment. However, the available evidence does suggest better outcomes for patients treated with a later generation quinolone, ethionamide or prothionamide, four or more effective drugs in the intensive phase, and three or more effective drugs in the continuation phase.$^{26}$ Extensively drug resistant tuberculosis treatment is even more challenging and often relies on drugs with only in vitro or animal data to support their use.

\section{Emerging treatments}

For the first time in over 40 years new antituberculosis drugs are becoming available; notably delamanid and bedaquiline, both of which already have a compassionate use role in difficult to treat multidrug resistant tuberculosis and extensively drug resistant tuberculosis. Furthermore there is renewed interest in the efficacy of clofazamine and linezolid. However, an urgent need remains for better regimens rather than new single drugs, and for this reason a handful of phase III trials are in progress. Promising results have been reported with the use of a nine month regimen in Bangladesh and several West African countries, which is now being formally tested in a randomised controlled trial. ${ }^{27}$ One view is that some regimens under investigation may ultimately be effective for all strains of tuberculosis whether or not susceptible to rifampicin or isoniazid, which would considerably attenuate the importance of the notion of multidrug resistant tuberculosis in global tuberculosis.

\section{What is the role of palliative care?}

Some patients with multidrug resistant tuberculosis will, despite apparently adequate treatment, fail to respond and require palliative care. This is particularly a problem for patients with 


\section{Box 2 Example of how drug side effects can affect treatment}

After the symptoms of a 26 year old Swazi woman failed to respond to two months of first line tuberculosis treatment (isoniazid, rifampicin, ethambutol, pyrazinamide) she underwent drug susceptibility testing and was found to have multidrug resistant tuberculosis. Treatment was started with moxifloxacin, kanamycin, ethionamide, and cycloserine, and she continued with pyrazinamide. Early substitution of cycloserine, to which her unbearable initial low mood and irritability was attributed, with para-aminosalicylate sodium replaced neuropsychiatric side effects with profuse diarrhoea. The onset of aminoglycoside induced hearing loss and the prospect of going deaf led to the patient discontinuing treatment after completing only four of 18 months of the regimen.

extensively drug resistant tuberculosis. Moreover, there are increasing reports of patients with no treatment options. Only 11 countries with a high burden of multidrug resistant tuberculosis (South Africa the only one outside Europe) reported palliative care facilities as part of their national tuberculosis plan. ${ }^{1}$ In high burden countries such as South Africa, where resources for home based palliative care are limited, it has even been suggested that modern day sanatoriums may provide access to palliative care on a large scale, while potentially reducing onward transmission..$^{28}$ However this approach has drawn criticism in equal measure, in part because the impact of reducing onward transmission by that stage is likely to be small, and because of potential risks from worsening stigma. ${ }^{29}$

\section{What are the public health implications?}

In addition to the complexities of individual case management, there are major public health implications to multidrug resistant tuberculosis. Most transmission is likely to occur before treatment and hence earlier diagnosis is a key determinant in the control of multidrug resistant tuberculosis. There is unlikely to be sufficient bed capacity for full scale-up of hospital based treatment for multidrug resistant tuberculosis and there is increasing evidence for ambulatory, community based treatment that may also reduce the risk of transmission within healthcare settings. Where inpatient admission is required, triage systems, strict infection control practices, building design for improved ventilation (including natural ventilation), and upper room (away from patients and staff) germicidal ultraviolet light all have an important role in reducing transmission. Moreover, in other key transmission hotspots (for example, prisons) there is a need for active intervention to identify cases and reduce transmission.

\section{What are the future directions?}

Until relatively recently few national, international, or non-governmental organisations had prioritised multidrug resistant tuberculosis, with a focus instead on drug susceptible tuberculosis. ${ }^{6}$ Now multidrug resistant tuberculosis forms a key part in the STOP TB Global Plan to Stop TB 2011-15 $5^{30}$ and has garnered attention at the highest international levels with a World Health Assembly resolution (WHA 62.15) calling on WHO members to provide universal access to multidrug resistant tuberculosis treatment. The STOP TB target is for all patients with newly diagnosed tuberculosis at high risk for multidrug resistance and all previously treated patients to undergo drug susceptibility testing. ${ }^{30}$ Achieving global control of multidrug resistant tuberculosis requires timely identification of cases, enrolment into quality assured treatment with relatively short, inexpensive, non-toxic treatment, minimisation of transmission, and accurate epidemiological surveillance. Unfortunately, there are major challenges to achieving each of these steps.

Contributors: All authors contributed equally to the writing and reviewing of all versions of this manuscript. DAJM is the guarantor.

Competing interests: We have read and understood the BMJ policy on declaration of interests and declare the following interests: none.

Provenance and peer review: Commissioned; externally peer reviewed.
1. World Health Organization. Global tuberculosis report 2014. WHO, 2014

2 Sullivan T, Ben Amor Y. What's in a name? The future of drug-resistant tuberculosis classification. Lancet Infect Dis 2013;13:373-6.

3 Anderson LF, Tamne S, Brown T, Watson J, Mullarkey C, Zenner D, et al. Transmission of multidrug-resistant tuberculosis in the UK: a cross-sectional molecular and epidemiological study of clustering and contact tracing. Lancet Infect Dis 2014:14:406-15.

4 Furin J, Bayona J, Becerra M, Farmer P, Golubkov A, Hurtado R, et al. Programmatic management of multidrug-resistant tuberculosis: models from three countries. Int $J$ Tuberc Lung Dis 2011;15:1294-300.

5 Health Protection Agency. Tuberculosis in the UK 2013 report. HPA, 2013.

6 Keshavjee S, Farmer PE. Tuberculosis, drug resistance, and the history of modern medicine. N Engl J Med 2012;367:931-6.

7 Nardell E, Dharmadhikari A. Turning off the spigot: reducing drug-resistant tuberculosis transmission in resource-limited settings. Int J Tuberc Lung Dis 2010;14:1233-43.

8 Caminero JA. Multidrug-resistant tuberculosis: epidemiology, risk factors and case finding. Int J Tuberc Lung Dis 2010;14:382-90

9 Faustini A, Hall AJ, Perucci CA. Risk factors for multidrug resistant tuberculosis in Europe: a systematic review. Thorax 2006;61:158-63.

10 Peter JG, Theron G, Pooran A, Thomas J, Pascoe M, Dheda K. Comparison of two methods for acquisition of sputum samples for diagnosis of suspected tuberculosis in smear-negative or sputum-scarce people: a randomised controlled trial. Lancet Resp Med 2013;1:471-8.

11 Martin A, Panaiotov S, Portaels F, Hoffner S, Palomino JC, Angeby K. The nitrate reductase assay for the rapid detection of isoniazid and rifampicin resistance in Mycobacterium tuberculosis : a systematic review and meta-analysis. $J$ Antimicrob Chemother 2008;62:56-64.

12 Moore DA, Evans CA, Gilman RH, Caviedes L, Coronel J, Vivar A. Microscopic-observation drug-susceptibility assay for the diagnosis of TB. N Engl J Med 2006;355:1539-50.

13 World Health Organization. Noncommercial culture and drug-susceptibility testing methods for screening patients at risk for multidrug-resistant tuberculosis: policy statement. WHO, 2011.

14 World Health Organization. Automated real-time nucleic acid amplification technology for rapid and simultaneous detection of tuberculosis and rifampicin resistance: Xpert MTB/RIF assay for the diagnosis of pulmonary and extrapulmonary TB in adults and children. Policy update. WHO, 2013.

15 Somoskovi A, Deggim V, Ciardo D, Bloemberg GV. Diagnostic implications of inconsisten results obtained with the Xpert MTB/Rif assay in detection of Mycobacterium tuberculosis isolates with an rpoB mutation associated with low-level rifampin resistance. J Clin Microbiol 2013;51:3127-9.

16 Zetola NM, Shin SS, Tumedi KA, Moeti K, Ncube R, Nicol M, et al. Mixed Mycobacterium tuberculosis complex infections and false-negative results for rifampin resistance by GeneXpert MTB/RIF are associated with poor clinical outcomes. J Clin Microbiol 2014;52:2422-9.

17 Choi HW, Miele K, Dowdy D, Shah M. Cost-effectiveness of Xpert(R) MTB/RIF for diagnosing pulmonary tuberculosis in the United States. Int $J$ Tuberc Lung Dis 2013;17(10):1328-35

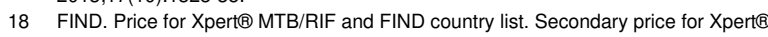
MTB/RIF and FIND country list 2013. www.finddiagnostics.org/about/what we do/ successes/find-negotiated-prices/xpert_mtb_rif.html.

19 World Health Organization. Tuberculosis diagnostics fact sheet. Secondary tuberculosis diagnostics fact sheet. www.who.int/tb/publications/tbDiagnostics_factsheet.pdf?ua=1.

20 Hillemann D, Rusch-Gerdes S, Richter E. Evaluation of the GenoType MTBDRplus assay for rifampin and isoniazid susceptibility testing of Mycobacterium tuberculosis strains and clinical specimens. J Clin Microbiol 2007;45:2635-40.

21 Raizada N, Sachdeva KS, Chauhan DS, Malhotra B, Reddy K, Dave PV, et al. A multi-site validation in India of the line probe assay for the rapid diagnosis of multi-drug resistant tuberculosis directly from sputum specimens. PLoS One 2014;9:e88626.

22 Barnard M, Gey van Pittius NC, van Helden PD, Bosman M, Coetzee G, Warren RM. The diagnostic performance of the GenoType MTBDRplus version 2 line probe assay is equivalent to that of the Xpert MTB/RIF assay. J Clin Microbio/ 2012;50:3712-6.

23 Koser CU, Bryant JM, Becq J, Török ME, Ellington MJ, Marti-Renom MA, et al. Whole-genome sequencing for rapid susceptibility testing of $M$. tuberculosis. N Engl J Med 2013;369:290-2

24 Chiang CY, Van Deun A, Enarson DA. A poor drug-resistant tuberculosis programme is worse than no programme: time for a change. Int J Tuberc Lung Dis 2013;17:714-8.

25 World Health Organization. Guidelines for the programmatic management of drug-resistan tuberculosis 2011. http://whqlibdoc.who.int/publications/2011/9789241501583 eng.pdf.

26 Ahuja SD, Ashkin D, Avendano M, Banerjee R, Bauer M, Bayona JN, et al. Multidrug resistant pulmonary tuberculosis treatment regimens and patient outcomes: an individual patient data meta-analysis of 9,153 patients. PLOS Med 2012;9:e1001300.

27 Van Deun A, Maug AK, Salim MA, Das PK, Sarker MR, Daru P, et al. Short, highly effective, and inexpensive standardized treatment of multidrug-resistant tuberculosis. $A m$ J Resp Crit Care Med 2010;182:684-92.

28 Dheda K, Migliori GB. The global rise of extensively drug-resistant tuberculosis: is the time to bring back sanatoria now overdue? Lancet 2012;379:773-5.

29 Hughes J, Cox H, Ford N. Sanatoria for drug-resistant tuberculosis: an outdated response. Lancet 2012;379:2148.

30 Stop TB Partnership. The global plan to stop TB 2011-2015: transforming the fight towards elimination of tuberculosis. WHO, 2010. 


\section{Additional educational resources}

Resources for healthcare professionals

World Health Organization. Multidrug resistant tuberculosis website (www.who.int/tb/challenges/mdr/en/)—Contains several resources, including clinical and programmatic guidelines

Lancet Infectious Diseases March-June 2013-Series of papers covering key areas in tuberculosis, with particular relevance for multidrug resistant tuberculosis

\section{Resources for patients}

Centres for Disease Control. Tuberculosis fact sheet (www.cdc.gov/tb/publications/factsheets/drtb/mdrtb.htm)—Provides information on multidrug resistant tuberculosis 


\section{Table}

Table 1| Incidence of tuberculosis and multidrug resistant tuberculosis in selected countries in 2013

\begin{tabular}{|c|c|c|c|c|}
\hline \multirow[t]{2}{*}{ Selected countries } & \multicolumn{2}{|c|}{ No of new cases } & \multicolumn{2}{|c|}{$\%(95 \% \mathrm{Cl})$ of cases with multidrug resistant tuberculosis } \\
\hline & Tuberculosis & $\begin{array}{l}\text { Multidrug resistant } \\
\text { tuberculosis }\end{array}$ & Newly diagnosed & Retreatment \\
\hline \multicolumn{5}{|c|}{ High burden, ${ }^{*}$ high resistance: $†$} \\
\hline Russian Federation & 130000 & 41000 & 19 (14 to 25$)$ & 49 (40 to 59$)$ \\
\hline China & 980000 & 542000 & $5.7(4.5$ to 7$)$ & 26 (22 to 30$)$ \\
\hline India & 2100000 & 61000 & $2.2(1.9$ to 2.6$)$ & $15(11$ to 19$)$ \\
\hline South Africa & 450000 & 6800 & $1.8(1.4$ to 2.3$)$ & 6.7 (5.4 to 8.2$)$ \\
\hline \multicolumn{5}{|c|}{ Non-high burden, high resistance: $†$} \\
\hline Belarus & 65000 & 1790 & 35 (33 to 37$)$ & 55 (52 to 57$)$ \\
\hline Ukraine & 44000 & 9400 & $14(14$ to 15$)$ & 32 (31 to 33 ) \\
\hline \multicolumn{5}{|c|}{ Non-high burden, non-high resistance: } \\
\hline Peru & 38000 & 2050 & 3.9 (3.6 to 4.2$)$ & 35 (33 to 37 ) \\
\hline United Kingdom & 8300 & 69 & $1.4(1$ to 1.7$)$ & 5.7 (3.2 to 9.4$)$ \\
\hline United States & 11000 & 108 & $1.2(0.98$ to 1.6$)$ & $3.9(1.9$ to 7.1$)$ \\
\hline
\end{tabular}

*22 Countries with highest number of cases of tuberculosis.

†27 Countries with highest number of cases of multidrug resistant tuberculosis. 